\title{
Time-resolved studies of Interatomic Coulombic Decay
}

\author{
U. Frühling ${ }^{1}$, F. Trinter ${ }^{2}$, F. Karimi ${ }^{1}$, J. B. Williams ${ }^{2}$, and T. Jahnke ${ }^{2 *}$ \\ 1 Institut für Experimentalphysik, \\ Universität Hamburg and Center for Ultrafast Imaging, \\ Luruper Chaussee 149, 22761 Hamburg, Germany \\ 2 Institut für Kernphysik, Goethe Universität, \\ Max-von-Laue-Str.1, 60438 Frankfurt, Germany
}

(Dated: June 9, 2015)

\begin{abstract}
Interatomic Coulombic Decay (ICD) is a decay mechanism occurring in loosely bound matter, e.g. in systems bound by van der Waals-forces or hydrogen bonds. In many such cases the decay time is similar to the time scale of nuclear motion during the decay. As the efficiency of ICD strongly depends on the internuclear distance of the atoms or molecules involved in the decay, an overall non-trivial temporal decay behavior arises. The progress of examining the time-domain aspects of Interatomic Coulombic Decay is summarized in this short topical review with a special emphasis on experiments that are now feasible due to the developments of free-electron lasers.
\end{abstract}

\section{INTRODUCTION}

Time-resolved studies typically have a particularly intriguing appeal, as they give in many cases intuitive and comprehensive insight into to the dynamics of atomic or molecular processes. Many techniques to access the time domain of the microscopic, atomic level have been employed during the last decades. While high resolution energy spectroscopy is often used to identify atomic or molecular states, it unveils (in many cases) information from the time domain, as well. The energy width of a decaying state is, for example, directly connected to its lifetime. Since the late nineteen eighties laser pumpprobe experiments explored the temporal evolution of the energy surfaces of bound molecular states on the femtosecond time scale [1]. Time-resolved electron diffraction enables observation of geometrical changes with sub picosecond resolution [2] and time-resolved X-ray diffraction is the key rationale for X-Ray free-electron lasers being built around the world. The shortest time scales down to the attosecond regime are typically accessed not by pump-probe measurements, but by using the concept of streaking. Just as in the case of a classic streak camera, a time dependent electric field is used to add momentum to an electron [3]: the electron momentum is then measured, enabling the time evolution of an atomic process to be determined. In a pioneering experiment of this type an attosecond VUV-pulse, created by high harmonic generation (HHG) by employing an $800 \mathrm{~nm}$ laser pulse, was used to eject a core-electron from Krypton atoms[4]. The $800 \mathrm{~nm}$ laser pulse was furthermore used to streak the subsequently emitted Auger electrons. Different versions of this technique were used in ground breaking experiments, which for example studied in a time-resolved manner the photoelectric effect in atoms [5], obtained information about tunneling times $[6,7]$, and monitored

\footnotetext{
*Electronic address: jahnke@atom.uni-frankfurt.de
}

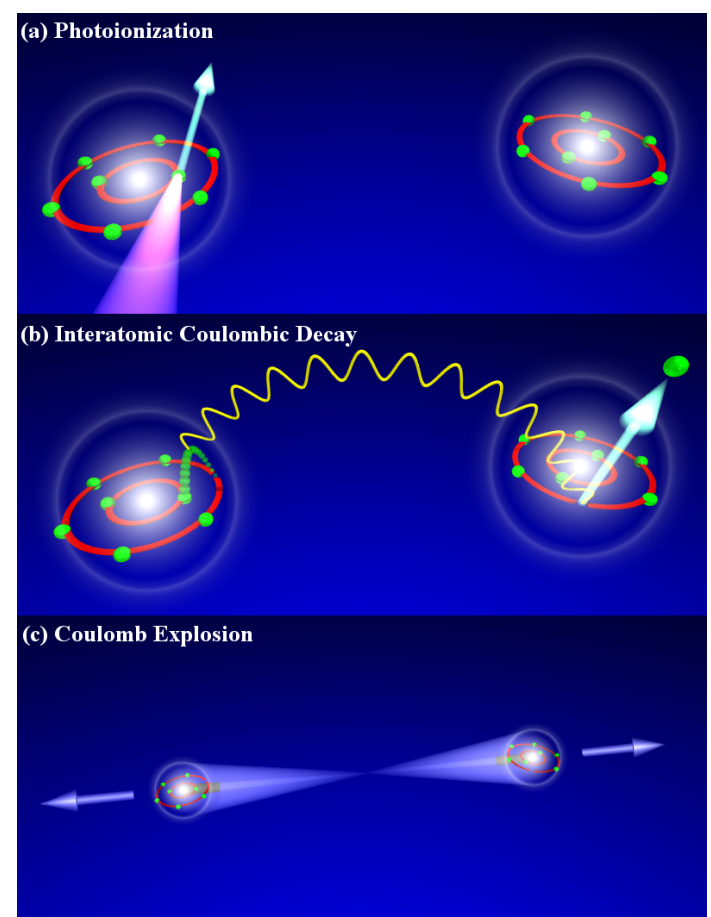

FIG. 1: Interatomic Coulombic Decay in a neon dimer: (a) A $2 s$-electron is removed from the left atom of a neon dimer. (b) As the vacancy is filled by a $2 p$-electron the energy gained is transferred to the right atom of the dimer. (c) Two singly charged neon ions are facing each other after the decay, thus causing the dimer to fragment in a Coulomb explosion. The figure was taken from [10].

electron emission from surfaces [8].

Being finally able to directly examine an Auger decay in a time-resolving experiment demonstrated incredible skills and progress in experimental techniques [4]. The defacto gain of atomic physics knowledge due to these impressive experiments was, however, limited, as the decay function of an Auger decay is strictly exponential 
and the corresponding decay width was already known for a long time from spectroscopy experiments. A decay phenomenon that has a more complex decay behavior, as compared e.g. to an Auger decay, is Interatomic (or Intermolecular) Coulombic Decay (ICD). ICD was predicted in 1997 by Cederbaum and coworkers [9] as a decay mechanism that occurs if an excited atom or molecule is embedded in a chemical environment. Typical scenarios where ICD takes place consist of loosely bound matter as van der Waals-Clusters or systems bound by hydrogen bonds. In such systems, an excitation energy can be transferred from an excited atom or molecule to a neighboring particle causing an emission of an electron from the particle receiving the energy. This electron is called an ICD electron and is typically of low kinetic energy. A sketch of ICD in a neon dimer is shown in Fig. 1 (taken from [10]). A photoionization of an inner-valence electron of the left atom of the dimer creates an excited state that will undergo ICD (Fig. 1a). As ICD occurs the right atom is ionized (Fig. 1b), finally causing the doubly charged dimer to fragment in a Coulomb-explosion (Fig. 1c). In the case of ICD, the decay probability depends strongly on the internuclear distance $R$ between the excited particle and the atom or molecule that emits the ICD electrons [11-15]. For large internuclear distances, for example, the main contribution to ICD scales with $1 / R^{6}[16]$. Already early theoretical investigations on ICD in neon dimers examined in high detail the dependence of the decay width $\Gamma$ on the internuclear distance as shown in Fig. 2 [11]. The key ingredient to a complex decay behavior is the typical time scale of ICD: the decay occurs on the same time scale as that of nuclear motion. When photoionization occurs nuclear motion is initiated, since in a typical scenario the excited state consists of a slightly attractive potential energy surface (i.e. the dimer starts to shrink). Therefore the dependence of the decay width on the internuclear distance has a large impact already on elementary features, such as the ICD electron energy spectrum or the kinetic energy release (KER) of the emitted ions. Additionally, it is obvious, that ICD became a prime candidate for a time-resolved examination: already the decay function was expected to show a distinct non-exponential behavior and as the vibrational wave packet of the decaying state evolves during the decay, observing its temporal evolution seemed intriguing.

\section{A. Early theoretical and experimental work}

Already one of the three pioneering publications that demonstrated in an experiment that ICD exists $[10,17$, 18] investigated temporal aspects of the decay. Öhrwall et al. determined the lifetime of a $2 s$ vacancy in neon clusters [18]. They found that in contrast to neon monomers, where the $2 s$ vacancy has a lifetime of 150 picoseconds [19], the lifetime reduces to a few femtoseconds in the case of neon clusters. The experimental results are shown in Fig. 3. The contribution from non-condensed

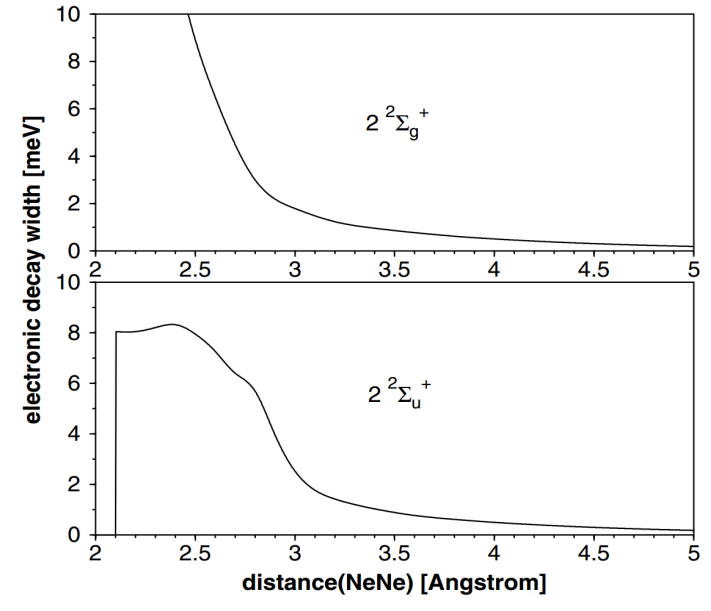

FIG. 2: The decay width of the two inner-valence states of the neon dimer as a function of the internuclear distance. The figure was taken from [11].

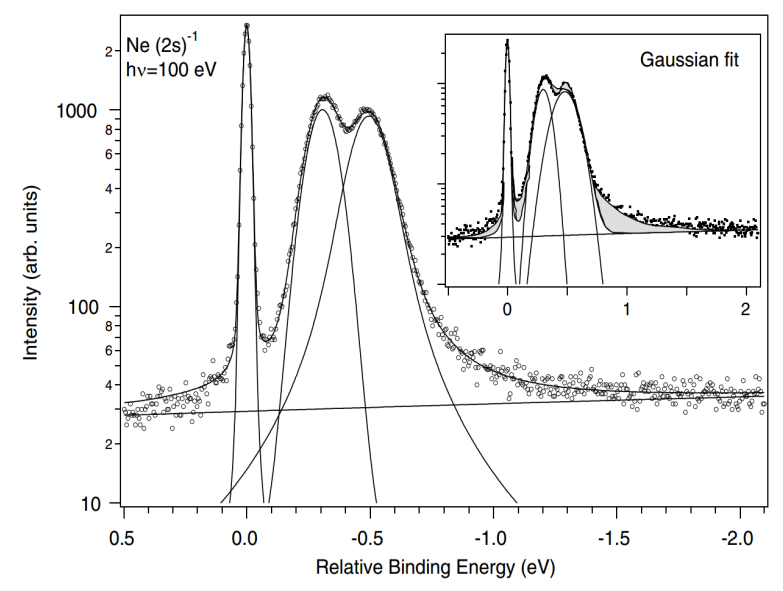

FIG. 3: Main figure: Voigt-fit to the $\mathrm{Ne}\left(2 s^{-1}\right)$ photoelectron spectrum recorded at $h \nu=100 \mathrm{eV}$. Inset: fit employing a Gaussian line profile. The cluster size is $\langle N\rangle \approx 900$ in both cases. The peak at $0 \mathrm{eV}$ belongs to the atomic (i.e. uncondensed) fraction of the Ne cluster beam displaying the instrumental energy resolution of $30 \mathrm{meV}$. (Figure taken from [18].)

neon atoms is located at a relative binding energy of $0 \mathrm{eV}$. It occurs as a Gaussian line shape with a spectral width of $30 \mathrm{meV}$, which is due to the instrumental broadening. The features that were attributed to bulk and surface atoms of the clusters, however, show a substantial lifetime broadening. Employing a Voigt-fit, the $2 s$ hole lifetime for bulk atoms was found to be $6 \pm 1 \mathrm{fs}$, while atoms being located at the surface of the cluster decay on average after times longer than $30 \mathrm{fs}$.

These findings were in line with earlier predictions by Santra et al. [20] that elucidated the effect of increasing the number of nearest neighbors to an inner-valence 


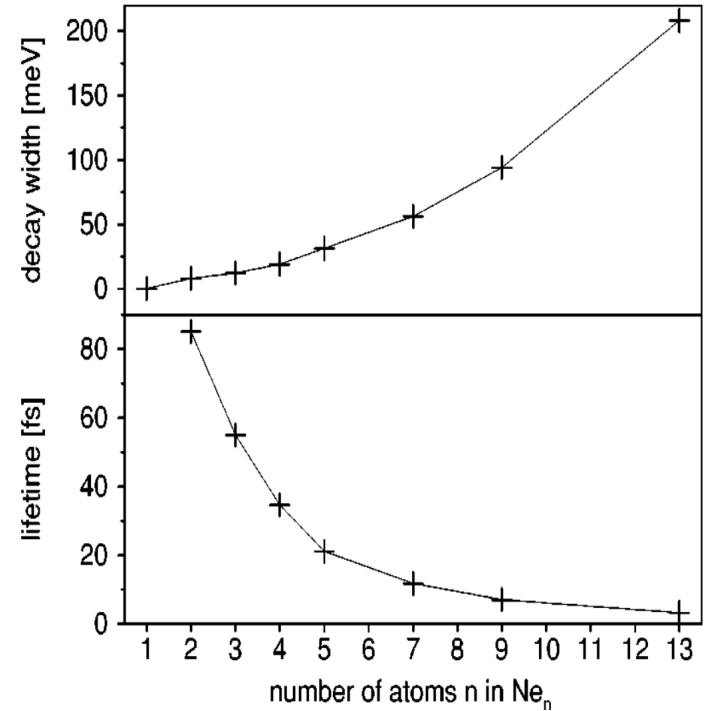

FIG. 4: Electronic decay width (top) and lifetime (bottom) of an inner-valence vacancy in $\mathrm{Ne}_{n}$. The figure was taken from [20].

ionized neon monomer. It was found that the lifetime of the vacancy decreases with increasing size of the cluster due to the increasing number of open decay channels (i.e. "decay partners"). While a $2 s$ vacancy in a neon dimer decays within $85 \mathrm{fs}$, the lifetime of a $2 s$ hole in larger clusters of size $n=13$ goes down to approx. 3 fs. Fig. 4 shows the calculated decay width for clusters $\mathrm{Ne}_{n}$ of different size $n$. More recent theory work on this issue has been published, e.g. in [21, 22].

A similar approach to that of Öhrwall et al. [18] was recently chosen by Trinter and coworkers [23]. In their experimental work they investigated ICD after resonant excitation of a HeNe mixed cluster [24-31]: a resonant excitation of the $\mathrm{He}$ atom caused the $\mathrm{Ne}$ atom to be ionized after ICD. The amount of resulting $\mathrm{HeNe}^{+}$was measured while scanning the photon energy of the exciting synchrotron beam from below to slightly above the threshold of the He-resonance. This absorbtion measurement yielded vibrationally resolved decay widths of ICD of the different vibrational states of the HeNe dimer. The lifetimes obtained depend on the exact vibrational state populated and are in the range of 100 fs to 1 ps. A detailed theoretical description can be found in [32].

Theoretical investigations of ICD in NeAr dimers after K - L L Auger decay of the Ne atom suggested another potential possibility to measure the decay time in an experiment [33]. Detailed analysis of the possible decay pathways identified a decay from $\mathrm{Ne}^{2+}\left(2 s^{-1} 2 p^{-1}{ }^{3} \mathrm{P}\right) \mathrm{Ar}$ into the manifold of $\mathrm{Ne}^{2+}\left(2 p^{-2}{ }^{3} \mathrm{P}\right)-\mathrm{Ar}^{1+}\left(3 p^{-1}\right)$ final states. As the decay of the triplet state is comparably slow (estimations of 80 fs were given), the decay time is for these states again in the same range as typical times of nuclear dynamics. The computed ICD electron spectra showed a broadened, asymmetric distribution with a shoulder on the low energy side, which was attributed to the nuclear motion occurring during the decay. In 2011 these predictions were confirmed by coincidence measurements by Ouchi et al. [34], that observed similar features in the KER-spectrum of that decay path.

Pokapanich et al. demonstrated a further experimental approach to obtain timing information on ICD of aqueous ions [35]. A so called "core-hole clock" was employed by measuring the ratio of occurring ICD and competing Auger decay. As the Auger lifetimes are known, the decay times of ICD can be extracted from these ratios.

Employing a similar concept of quenching ICD, the decay times of highly excited states of $\mathrm{N}_{2}$ and $\mathrm{CO}$ dimers were determined in 2013 [36]. These excited states were created after resonant core excitation and subsequent (single-site) spectator Auger decay. As it is known that the potential energy surfaces of these states are typically steeply repulsive (see e.g. [37]) two competing scenarios arise: firstly, the excited molecule dissociates, thus prohibiting ICD to occur or, secondly, ICD outpaces the dissociation stabilizing the molecule. As ICD was identified in the coincidence measurement, it was shown, that ICD occurs prior to a possible dissociation, i.e. on a timescale of approx. 12 fs.

\section{TIME-RESOLVING SYNCHROTRON MEASUREMENTS EMPLOYING POST COLLISION INTERACTION}

All the experimental work mentioned in the previous sections investigated only very crudely the time-domain aspects of ICD: a mean lifetime - implicitly assuming an exponential decay - was deduced from these measurements. The complex decay behavior predicted by theory was not accessible by these experiments. An experiment by Trinter et al. was recently able to follow the temporal evolution of the kinetic energy release distribution as a helium dimer IC-decayed after shakeup ionization [38] and to measure the non-exponential decay function of ICD.

As described in the introduction, a well established approach to measure the temporal evolution of a decaying system can be found in so called "streaking" experiments (see sect. III B). In this approach the atomic-scale timing information is typically mapped to a kinetic energy which can be measured. During recent years it was realized, that a corresponding mapping of decay time to photoelectron energy naturally occurs, whenever a decay produces a secondary electron, which is significantly faster than the photoelectron [39-41]. The influence of the Coulomb field of the second, fast electron on the previously emitted slow electron is termed "Post Collision Interaction" (PCI) [42]. This effect has been known for a long time, but so far mainly its energy domain aspects were considered [42-44]. It has been studied in great detail e.g. after Auger decay [43]. From a classical point of view PCI can be described as follows: as an Auger 
electron emission happens while a photoelectron emerges from its parent ion, the charge of the residual ion changes from single to double. Accordingly, the emerging photoelectron is suddenly exposed to the $2 / \mathrm{R}$ Coulomb field of a doubly charged ion instead of the $1 / \mathrm{R}$ potential of a single charge. As a consequence the photoelectron is decelerated (and the Auger electron is accelerated).

A link of PCI (as an energy regime effect) and the time domain is given by the fact, that the energy difference between a $1 / R$ and a $2 / R$ potential scales with $1 / R$. Therefore, again in a classical picture, the measured photoelectron energy $E_{P C I}$ is given by the initial photoelectron energy $E_{p}$ and the distance $R_{p}$ the photoelectron traveled prior to the decay:

$$
E_{P C I}=E_{p}-1 / R_{p}
$$

In this picture, the emitted Auger electron (after being ejected at the time $t_{A}$ ) needs to "overtake" the photoelectron for PCI to occur. This happens at the time $t_{O V R}=t_{A} v_{A} /\left(v_{A}-v_{p}\right)$ (with the velocity of the Auger electron $v_{A}$ and of the photoelectron $v_{p}$ ). This, finally, results in:

$$
E_{P C I}=E_{p}-\frac{v_{A}-v_{p}}{v_{p} v_{A} t_{A}}
$$

Therefore, as the decrease of the electron energy is an observable, an access to the time of single electronic decay events in an experiment arises. In the experiment described in [38] the ICD electron takes the place of the Auger electron. In Fig. 5a) the conversion function mapping a measured photoelectron kinetic energy to decay time is plotted. In the simulation an electron of kinetic energy of $\mathrm{E}_{p}=140 \mathrm{meV}$ is emitted. After a delay time the ICD electron, with a kinetic energy of 10 $\mathrm{eV}$ (which is the most typical kinetic energy for an ICD electron in the employed target system $\mathrm{He}_{2}$ ) is launched. It turns out, that the range of accessible decay times and in particular the minimum accessible decay time is determined by the initial energy of the photoelectron prior to PCI. This is due to the fact that electrons experiencing a strong decelaration may be recaptured into the ion [45]. As the initial photoelectron energy $E_{p}$ is increased, the minimum accessible decay time decreases. Fig. 5a) shows the conversion function for different values of $E_{p}$. Apart from being a fully classical model, it furthermore neglects effects that occur due to the emission angles of the two electrons. This effect is known to be strong only for a small region of almost equal emission directions, as shown by Landers et al. [44]. A significant deviation from a solid angle integrated model due to angular effects occurs within an angle of $\pm 20^{\circ}$ between the photoelectron and the Auger electron, which corresponds to $5.6 \%$ of the total $4 \pi$ solid angle. The inverse of this "PCI-streaking" concept was observed by Schütte et al. in 2012 [39]. In a time-resolved measurement of an Auger decay of Xe and

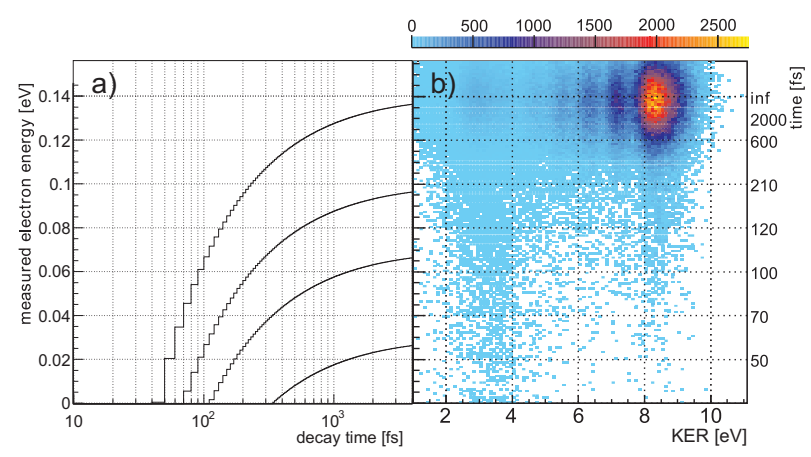

FIG. 5: (a) Conversion of measured photoelectron energy to decay time: the four lines show four examples of initial (undisturbed) photoelectron energies of (top to bottom) $140 \mathrm{meV}$, $100 \mathrm{meV}, 70 \mathrm{meV}$ and $30 \mathrm{meV}$ with the kinetic energy of the ICD electron being $10 \mathrm{eV}$. If, for example, an electron that is expected to occur at an energy of $140 \mathrm{meV}$ is decelerated due to PCI (see text) and measured at an energy of $60 \mathrm{meV}$ the corresponding decay time was 100 fs. (b) KER vs. measured electron energy. The time-axis on the right was obtained according to the most upper curve (initial energy of $140 \mathrm{meV}$ ). The figure was taken from [38].

Kr atoms, they observed a "chirp" of the Auger electron, i.e. a time-dependent shift of the Auger electron kinetic energy. These measurements (supported by further theoretical work [40]) accordingly confirm the validity of the PCI-streaking approach.

Trinter et al. employed Cold Target Recoil Ion Momentum Spectroscopy (COLTRIMS) [46-48] and PCIstreaking to measure the energy of the photoelectron carrying the time information and the momenta of the fragment ions occurring after ICD in coincidence. In an experimental setup similar to that described in [49] helium dimers were ionized and excited using linearly polarized synchrotron radiation of an energy of $65.536 \mathrm{eV}$ from beam line UE112-PGM-1 at BESSY. In Fig. 5b) the experimental results of the coincidence measurement are depicted showing the correlation of the measured kinetic energy release (KER) and the kinetic energy of the photoelectrons. As expected, the electron spectrum consists of a photoelectron line at an energy of approximately $140 \mathrm{meV}$ that is streaked towards lower electron energies. This figure reveals furthermore that the KER depends strongly on the time at which ICD takes place. For short decay times (i.e. low photoelectron energies) mainly lower KERs occur. For longer decay times a main peak at higher KER builds up and nodal features arise.

Within the so-called reflection approximation [50] a simple relation between the KER and the internuclear distance of the two atoms of the dimer at the instant of the Coulomb explosion of $\mathrm{KER}=1 / R$ (in atomic units) can be found. This leads to an intuitive picture: The helium dimer is a huge system [51] and large internuclear distances lead to small KER values. Thus, for short decay times a peak at lower kinetic energies occurs. As the 
nuclear motion sets in, the system starts to contract towards the mean internuclear distance of the excited ionic state, causing the main peak at $\mathrm{KER} \approx 8.5 \mathrm{eV}$ to build up. This KER value corresponds to the inner turning point of the vibrational motion of the ionized dimer. For the longest decay times, i.e. the smallest energy losses of the photoelectron, finally the previously observed vibrational features form, yielding the distribution, which is known from previous, non-time-resolved investigations $[49,52]$. By integrating over the kinetic energy release, finally, the decay function of ICD in the helium dimer was obtained experimentally and theoretically (see Fig. 6 ). It clearly shows the non-exponential decay behavior that occurs due to the nuclear dynamics during ICD. For the shortest decay times the decay rates are small as typical internuclear distances are large. As the dimer contracts during later decay times, the rates increase and keep showing the non-exponential behavior that is determined by nuclear motion. The experimental error bars are strongly asymmetric, especially at longer decay times due to the strongly non-linear conversion of the electron energy shift to decay time. The theory lines in Fig. 6 were calculated by Sisourat et al. studying the impact of nuclear dynamics on interatomic Coulombic decay in a He dimer [53]. The observed differences between the theoretical description and experiment are assumed to be attributed to the accuracy of the decay rates entering the nuclear dynamics simulation.

\section{TIME RESOLVED MEASUREMENTS AT FREE-ELECTRON LASERS}

In the PCI streaking experiments the interaction of the ICD- and photo-electron wave packets was used as a clock to extract the ICD lifetimes. This allows for measurements in the fs regime even though the ionizing light pulse from the synchrotron is much longer (approx. 50 ps) than the decay dynamics observed. In a more conventional approach, the process is triggered with a light pulse short compared to the investigated process and then a second light pulse impinging after a defined delay time is used to probe the system. The necessary ultrashort intense pulses in the XUV to soft X-ray range to perform ICD pump-probe experiments are now available at freeelectron lasers facilities $[54,55]$ and the intensities are sufficient even for XUV-pump and XUV-probe experiments as has recently been demonstrated. An experiment by the Heidelberg group employing a COLTRIMS setup [4648] and XUV pump-probe techniques was able to investigate the depletion of the decaying state in the time domain [56]. This state-of-the-art measurement performed at the Hamburg free-electron laser "FLASH" obtained a mean lifetime of decaying neon dimers of $150 \pm 50 \mathrm{fs}$, however, an exponential decay function was assumed in order to obtain this value. Combining the FEL-pulses with synchronized pulses of longer wavelengths will allow for a measurement of the IC-decay dynamics by streak-

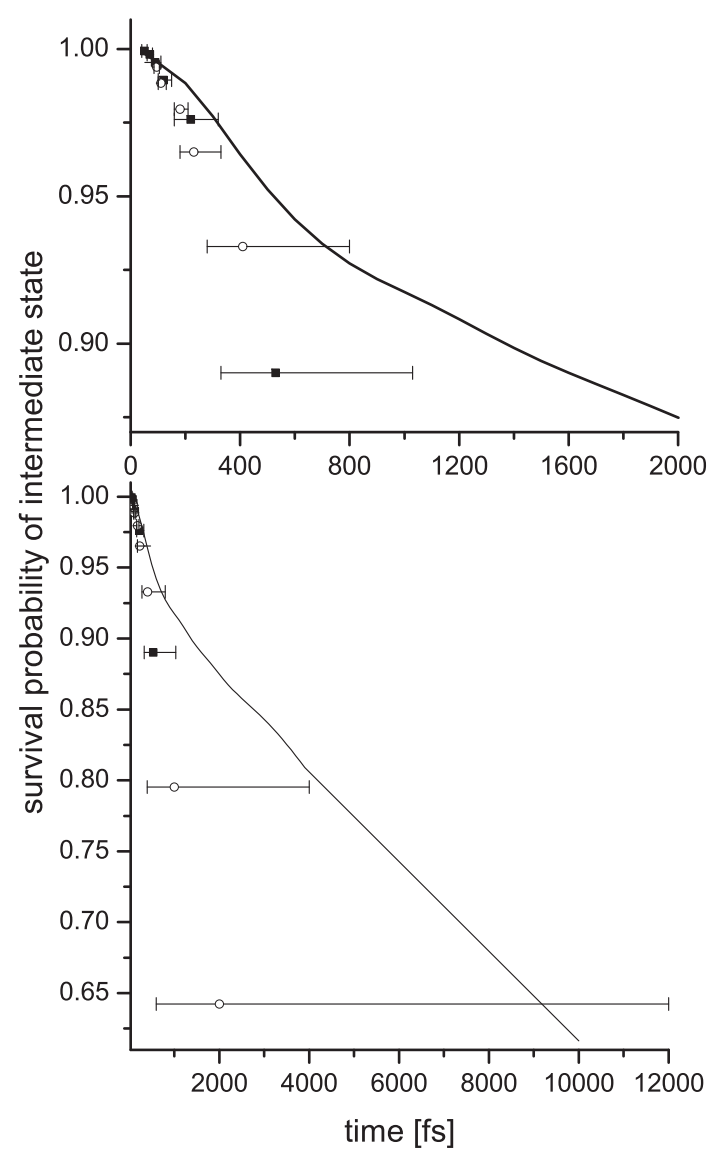

FIG. 6: Survival probability of the intermediate excited state that undergo ICD for two different time ranges as a function of time. The figure was taken from [38].

ing as has been proposed by several groups. The two techniques will be discussed in this section.

\section{A. XUV-pump/XUV-probe measurements}

Recently, Schnorr et al. [56] were able to perform a pump-probe measurement of ICD. They directly determined the lifetime of ICD in $\mathrm{Ne}_{2}$ employing a femtosecond extreme ultraviolet (XUV) high intensity pulse pair from the Hamburg free-electron laser (FLASH). The incoming light of $58.2 \mathrm{eV}$ was reflected off a multilayer split-and-delay mirror setup, splitting the beam into two halves with adjustable time delay in between. The two beam halves were focused into a supersonic gas jet of a COLTRIMS setup which was used to measure the momenta of the ions created by ICD in coincidence.

In order to trace the temporal features of ICD the following scheme was used: the pump pulse creates a $2 s$ inner-valence-shell vacancy in one of the two Ne atoms of a dimer and triggers the nuclear motion of the dimer ion (see Fig. 7). Subsequently, the vibrational wave packet propagates on the inner-valence ionized potential energy 


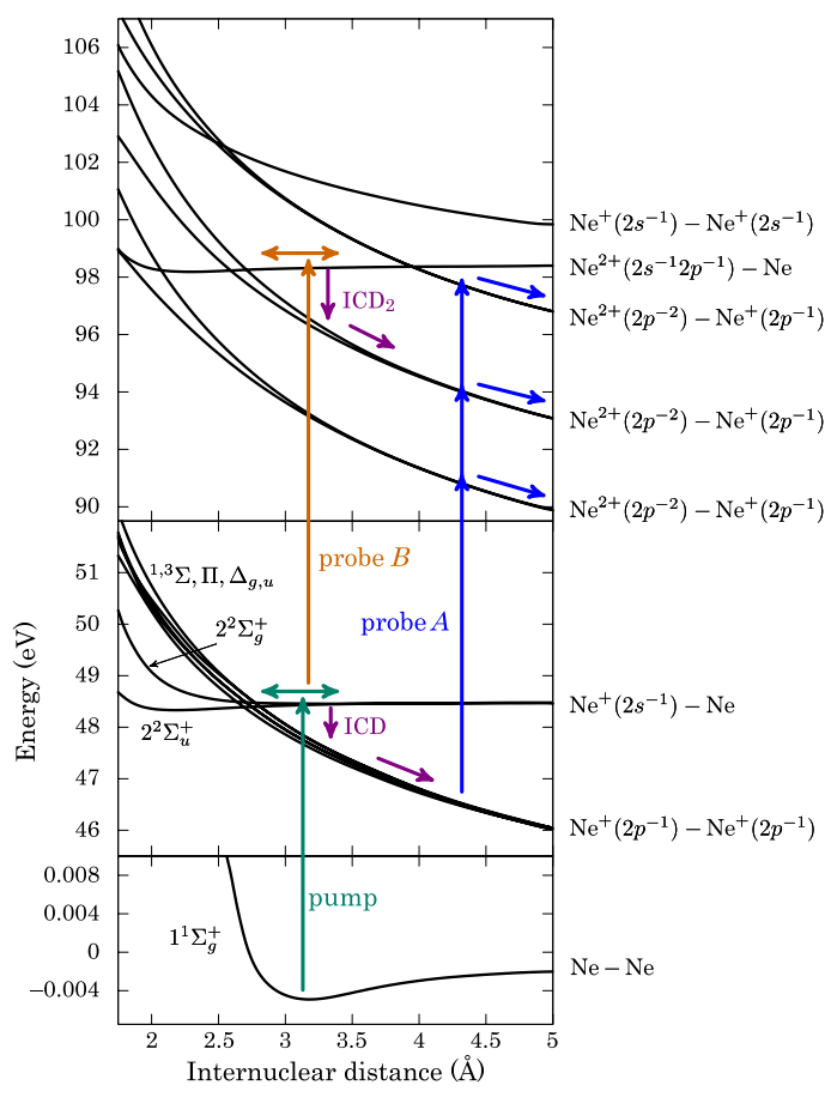

FIG. 7: Potential energy surfaces of the neon dimer that are involved in the pump-probe scheme by Schnorr et al. to extract the lifetime of single ICD events. (Figure taken from [56].)

curve until ICD occurs, resulting in a repulsive (Coulomb exploding) $\mathrm{Ne}^{+}\left(2 \mathrm{p}^{-1}\right)-\mathrm{Ne}^{+}\left(2 \mathrm{p}^{-1}\right)$ state. This state is probed by a second XUV-pulse, removing a further electron from one of the $\mathrm{Ne}^{+}$ions, and thus yielding triply charged ions in a $\mathrm{Ne}^{2+}\left(2 \mathrm{p}^{-2}\right)-\mathrm{Ne}^{+}\left(2 \mathrm{p}^{-1}\right)$ state. The triply ionized state is used as a fingerprint for the occurrence of ICD before the arrival of the probe pulse. As a function of the adjustable pump-probe delay, the yield of coincident $\mathrm{Ne}^{+}-\mathrm{Ne}^{2+}$ ion pairs is recorded, which is expected to increase with the time delay reflecting the ICD lifetime. In order to retrieve the mean decay time, a more detailed analysis, employing a classical simulation of the nuclear motion solving Newton's equation of motion for point-like particles, was performed. As an input to the simulation a constant lifetime and an exponential decay function were assumed. The KER for a particular time delay is obtained by adding up the kinetic energies that the particle has gained during its motion on the different potential energy curves occurring during the process. The simulation showed that other pathways which lead to the same final state (such as $\mathrm{ICD}_{2}$ (see Fig. 7), here $\mathrm{Ne}^{2+}\left(2 \mathrm{~s}^{-1} 2 \mathrm{p}^{-1}\right)$ - Ne undergoes ICD into $\left.\mathrm{Ne}^{2+}-\mathrm{Ne}^{+}\right)$ did not influence the calculation of the ICD lifetime. The

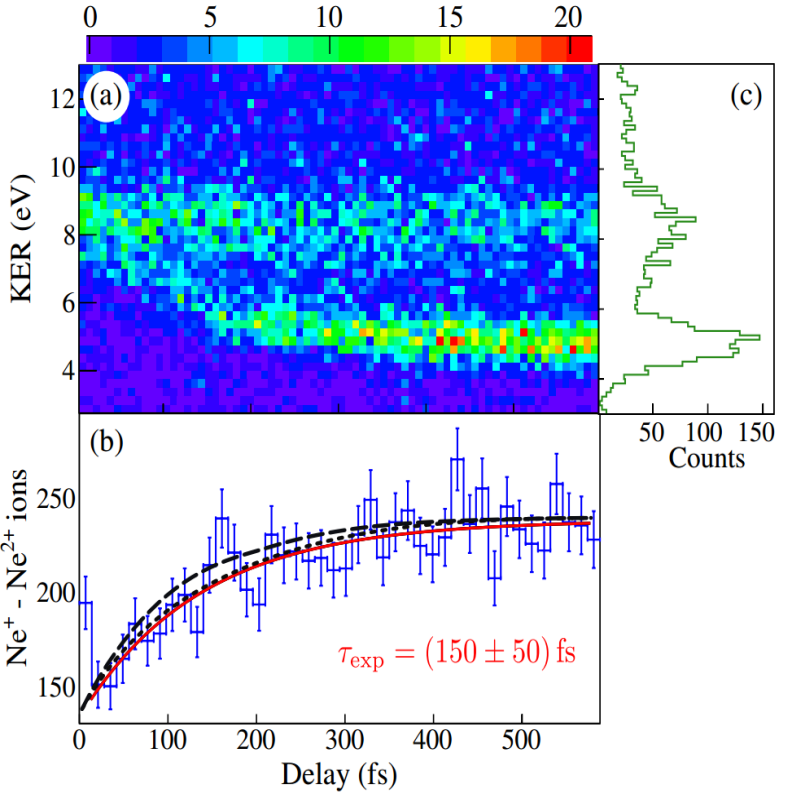

FIG. 8: (a) KER of measured breakups into $\mathrm{Ne}^{+} / \mathrm{Ne}^{2+}$ as a function of the pump-probe delay. (b) Dependence of the intensity of KERs between 3 and $11 \mathrm{eV}$ on the delay. The red line shows the exponential fit employed to retrieve the decay time, the black line corresponds to quantum mechanical calculations including nuclear dynamics. (c) KER for $400<$ $t_{\text {delay }}<600 \mathrm{fs}$. The figure was taken from [56].

latter was determined via an exponential fit of the projection of the measured KER versus delay distribution (shown in Fig. 8) onto the time delay axis. From this fit an ICD lifetime of $150 \pm 50$ fs was extracted. The error is purely statistical and the value gives an upper limit for the lifetime. This measured lifetime cannot be compared directly to computed ICD rates at a fixed internuclear distance or at a range of distances $[20,22,57,58]$. Instead, quantum mechanical calculations including the nuclear wave packet dynamics in the intermediate states must be performed, using the computational technique of [59]. The results of this impressive experiment compare well with these quantum calculations including nuclear dynamics, i.e. a $R$-dependent ICD width, as the black line in Fig. 8 b) shows.

\section{B. Employing THz-streaking to measure the decay dynamics of ICD}

Light-field streaking is a powerful method to measure ultrafast processes directly in the time domain whereby continuum electron wave packets are probed by lightfields. The temporal profiles of these wave packets carry the desired information about the underlying dynamics. The idea of the experiment is to initiate the process with an ultrashort (few femtosecond) XUV pulse and use a superimposed long wavelength light field which is phase 
locked to the ionizing light pulse to probe the continuum electron wave packet which is produced in the relaxation process by ICD. The emitted electrons will be accelerated by the streaking field whereby the resulting momentum change is proportional to the vector potential at the time of ionization (see Fig. 9). Thus the temporal profile of the electron wave packet is mapped onto electron momenta which can be measured. The resulting electron spectrum will then consist of a convolution of the streaked temporal profile and the unstreaked spectrum $[60,61]$. The probing light-field should be chosen such that the oscillation period is long compared to the duration of the electron wave packet dynamics and such that it is non-invasive, i.e. a photodissociation or other fieldinduced side-effects are avoided. In case of an oscillation period shorter than the electron wave packet quantum interferences between different portions of the ejected electron wave packet that experience the same momentum shift by the streak field, the probing light-field will cause the formation of spectral sidebands [4]. In such a case a direct time to energy mapping is no longer possible. For ICD lifetimes of a few tens to hundreds of fs that condition of a comparably long oscillation period is fulfilled for terahertz $(\mathrm{THz})$ radiation with wavelengths in the range of a few hundred $\mu \mathrm{m}$. Intense $\mathrm{THz}$ pulses suitable for streaking experiments can be generated at FLASH by a dedicated undulator setup [62]. Since the same electron bunches are used to generate both - the XUV and $\mathrm{THz}$ pulses - both pulses are inherently synchronized and phase locked. Alternatively the $\mathrm{THz}$ pulses can be generated by optical rectification of laser-pulses in crystals. Suitable synchronized lasers are available at all FEL facilities. THz streaking has at first been demonstrated at FLASH using the THz-undulator pulses [63]. Here it was possible to measure the temporal profile of fs photoelectron wave packets generated by the XUV pulses. It has later been repeated at a laboratory XUV source using a laser based THz-source [64] and as well at FELs using laser based $\mathrm{THz}$ sources [65].

As discussed above, the spectrum of ICD electrons is expected to be considerably influenced by nuclear motion. Since the equilibrium distance of the two atoms is shorter for the ionized system than for the neutral dimer the two atoms will move towards each other and oscillate after the ionization. This leads to a time dependency of the ICD electron spectrum. Early ICD electrons are emitted at a time when the internuclear distance is large and thus the ICD electron energy is high while for later ICD electrons the inter-nuclear distance can be shorter and the energy is thus lower. These temporal changes of the electron spectrum (i.e. a "chirp") can be determined by measuring streak-spectra under different angles with respect to the streak-field polarization and at different streak-field phases. This is depicted in Fig.10 where a linearly chirped electron wave packet is streaked with two opposite streak field directions. In the upper case the electron wave packet coincides with a rising streak vector potential. Here the slow electrons from the head

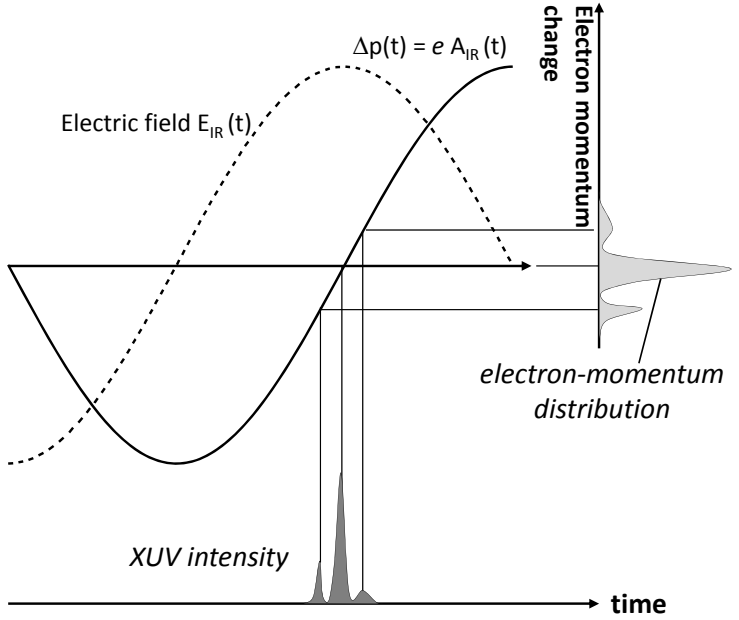

FIG. 9: Principle of light-field streaking. An electron wave packet is created by photoionization. The electrons are accelerated by the electric field of an infrared (IR) or THz streak pulse. The momentum change is given by the IR vector potential at the ionization time. Thus, the temporal profile of the electron wave packet is mapped onto changes of photoelectron momenta. (Figure taken from [66].)

of the wave packet are decelerated by the streak field while the fast electrons from the tail are further accelerated. This leads to a broad final electron spectrum. If the slope of the vector potential is reversed (lower case of Fig. 10) the electron spectrum will be narrower. By measuring streak spectra for both field directions and the non-streaked photoelectron spectrum the temporal profile and the first order of the chirp of the electron wave packet and thus the ICD dynamics can be reconstructed. These spectra can be measured simultaneously by using three photoelectron spectrometers or by using angle resolved electron spectrometers or alternatively by measuring spectra for different phases of the streaking field.

An intriguing approach can be found with the combination of FEL-THz streaking and a multi-coincidence measurement method as the COLTRIMS technique. In such an experiment the decay time for each decay event can be obtained by measuring the momenta of the (streaked) ICD electron and the two ions generated by the decay in coincidence. This is possible as the contribution of the streaking field to the overall momentum of the emitted ICD electron can be disentangled: the streaking field transfers a momentum to the electron and, correspondingly, to the center of mass of the dimer ion. For ICD the sum of the kinetic energy of the ICD electron and the KER is a constant. This fact was, for example, employed to identify ICD in the neon dimer [10]. As the streaking field does not affect the KER (as the KER is given by the relative momentum of the two ions gathered from the Coulomb explosion) any deviation from the expected sum kinetic energy is due to the streaking field. 


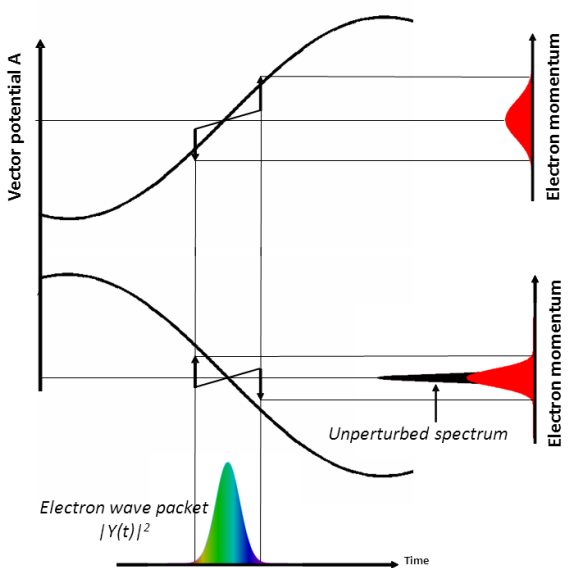

FIG. 10: Light-field streaking for chirped electron wave packets. In the upper case (rising slope of the streak vector potential) the slow electrons from the head of the wave packet are decelerated by the streaking field while the fast electrons from the tail are further accelerated. This leads to a broad final electron spectrum. In the lower case the situation is reversed.

This way the decay time of a single decay event can be measured, allowing for investigations of the temporal evo- lution of any other quantity obtained in the coincidence measurement, as e.g. the KER or angular emission distributions. A corresponding experiment has been proposed and will hopefully be carried out in the near future.

\section{SUMMARY}

ICD has been studied theoretically and experimentally since almost 20 years yielding a total of more than 200 publications on the topic, so far [67]. The temporal features of the decay have been of great interest since its first prediction. Most recently, first experiments were able to gather information which goes beyond the mean lifetimes. With the developments of FEL sources - and in particular - phase locked THz-pulse sources, further experiments in the time domain become feasible in the near future.

\section{ACKNOWLEDGMENTS}

This work was supported by the Deutsche Forschungsgemeinschaft (DFG) as part of the "DFGForschergruppe" FOR1789.
[1] A.H. Zewail. Femtochemistry: Ultrafast Dynamics of the Chemical Bond, volume I and II. World Scientific, Singapore (1994).

[2] G. Sciaini, M. Harb, S.G. Kruglik, T. Payer, C.T. Hebeisen, F.J.M. zu Heringdorf, M. Yamaguchi, M. Horn-von Hoegen, R. Ernstorfer, and R.J.D. Miller. Electronic acceleration of atomic motions and disordering in bismuth. Nature, 458, 7234 (2009).

[3] M.Ya. Schelev, M.C. Richardson, and A.J. Alcock. Image-Converter Streak Camera With Picoseond Resolution. Appl. Phys. Lett., 18, 354 (1971).

[4] M. Drescher, M. Hentschel, R. Kienberger, M. Uiberacker, V. Yakovlev, A. Scrinzi, Th. Westerwalbesloh, U. Kleineberg, U. Heinzmann, and F. Krausz. Timeresolved atomic inner-shell spectroscopy. Nature, 419, 803 (2002).

[5] M. Schultze, M. Fiess, N. Karpowicz, J. Gagnon, M. Korbman, M. Hofstetter, S. Neppl, A.L. Cavalieri, Y. Komninos, Th. Mercouris, C.A. Nicolaides, R. Pazourek, S. Nagele, J. Feist, J. Burgdörfer, A.M. Azzeer, R. Ernstorfer, R. Kienberger, U. Kleineberg, E. Goulielmakis, F. Krausz, and V.S. Yakovlev. Delay in Photoemission. Science, 328, 1658-1662 (2010).

[6] M. Uiberacker, Th. Uphues, M. Schultze, A.J. Verhoef, V. Yakovlev, M.F. Kling, J. Rauschenberger, N.M. Kabachnik, H. Schröder, M. Lezius, K.L. Kompa, H.G. Muller, M.J.J. Vrakking, S. Hendel, U. Kleineberg, U. Heinzmann, M. Drescher, and F. Krausz. Attosecond real-time observation of electron tunnelling in atoms. $\mathrm{Na}$ ture, 446, 627-632 (2007).

[7] P. Eckle, A. Pfeiffer, C. Cirelli, A. Staudte, R. Dörner,
H.G. Muller, M. Büttiker, U. Keller. Attosecond Ionization and Tunneling Delay Time Measurements in Helium. Science, 322, 1525 (2008).

[8] A.L. Cavalieri, N. Müller, Th. Uphues, V.S. Yakovlev, A. Baltus caronka, B. Horvath, B. Schmidt, L. Blümel, R. Holzwarth, S. Hendel, M. Drescher, U. Kleineberg, P.M. Echenique, R. Kienberger, F. Krausz, and U. Heinzmann. Attosecond spectroscopy in condensed matter. Nature, 449, 1029-1032 (2007).

[9] L.S. Cederbaum, J. Zobeley, and F. Tarantelli. Giant intermolecular decay and fragmentation of clusters. Phys. Rev. Lett., 79, 4778 (1997).

[10] T. Jahnke, A. Czasch, M.S. Schöffler, S. Schössler, A. Knapp, M. Käsz, J. Titze, C. Wimmer, K. Kreidi, R.E. Grisenti, A. Staudte, O. Jagutzki, U. Hergenhahn, H. Schmidt-Böcking, and R. Dörner. Experimental Observation of Interatomic Coulombic Decay in Neon Dimers. Phys. Rev. Lett., 93, 163401 (2004).

[11] R. Santra, J. Zobeley, and L.S. Cederbaum, N. Moiseyev. Interatomic Coulombic Decay in van der Waals Clusters and Impact of Nuclear Motion. Phys. Rev. Lett., 85, 4490 (2000).

[12] P. Kolorenc, V. Averbukh, K. Gokhberg, and L.S. Cederbaum. Ab initio calculation of interatomic decay rates of excited doubly ionized states in clusters. J. Chem. Phys., 129, 244102 (2008).

[13] S. Kopelke, K. Gokhberg, L.S. Cederbaum, and V. Averbukh. Calculation of resonant interatomic Coulombic decay widths of inner-valence-excited states delocalized due to inversion symmetry. J. Chem. Phys., 130, 144103 (2009). 
[14] K. Gokhberg, S. Kopelke, N.V. Kryzhevoi, P. Kolorenc, and L.S. Cederbaum. Dependence of interatomic decay widths on the symmetry of the decaying state: Analytical expressions and ab initio results. Phys. Rev. A, 81, 013417 (2010).

[15] S. Kopelke, K. Gokhberg, V. Averbukh, F. Tarantelli, and L.S. Cederbaum. Ab initio interatomic decay widths of excited states by applying Stieltjes imaging to Lanczos pseudospectra. J. Chem. Phys., 134, 094107 (2011).

[16] V. Averbukh, I.B. Müller, and L.S. Cederbaum Mechanism of Interatomic Coulombic Decay in Clusters. Phys. Rev. Lett., 93, 263002 (2004).

[17] S. Marburger, O. Kugeler, U. Hergenhahn, and T. Möller. Experimental evidence for Interatomic Coulombic decay in Ne clusters. Phys. Rev. Lett., 90, 203401 (2003).

[18] G. Öhrwall, M. Tchaplyguine, M. Lundwall, R. Feifel, H. Bergersen, T. Rander, A. Lindblad, J. Schulz, S. Peredkov, S. Barth, S. Marburger, U. Hergenhahn, S. Svensson, and O. Björneholm. Femtosecond Interatomic Coulombic Decay in Free Neon Clusters: Large Lifetime Differences between Surface and Bulk. Phys. Rev. Lett., 93, 173401 (2004)

[19] L.J. Curtis and Wm. Hayden Smith. Radiative-lifetime and absolute-oscillator-strength studies for some resonance transitions of Si I, II, and III. Phys. Rev. A, 9, 1537 (1974).

[20] R. Santra, J. Zobeley, and L.S. Cederbaum. Electronic decay of valence holes in clusters and condensed matter. Phys. Rev. B, 64, 245104 (2001).

[21] A. Ghosh and N. Vaval. Geometry-dependent lifetime of Interatomic coulombic decay using equation-of-motion coupled cluster method. J. Chem. Phys., 141, 234108 (2014).

[22] V. Averbukh and L.S. Cederbaum. Calculation of interatomic decay widths of vacancy states delocalized due to inversion symmetry. J. Chem. Phys., 125, 094107 (2006).

[23] F. Trinter, J.B. Williams, M. Weller, M. Waitz, M. Pitzer, J. Voigtsberger, C. Schober, G. Kastirke, C. Müller, C. Goihl, P. Burzynski, F. Wiegandt, R. Wallauer, A. Kalinin, L.Ph.H. Schmidt, M.S. Schöffler, Y.C. Chiang, T. Jahnke, and R. Dörner. Vibrationally Resolved Decay Width of Interatomic Coulombic Decay in HeNe. Phys. Rev. Lett., 111, 233004 (2013).

[24] K. Gokhberg, A.B. Trofimov, T. Sommerfeld, and L.S. Cederbaum. Ionization of metal atoms following valenceexcitation of neighbouring molecules. Europhys. Lett., 72, 228 (2005).

[25] S. Barth, S. Joshi, S. Marburger, V. Ulrich, A. Lindblad, G. Ohrwall, O. Bjorneholm, and U. Hergenhahn. Observation of resonant interatomic Coulombic decay in $\mathrm{Ne}$ clusters. J. Chem. Phys., 122, 241102 (2005).

[26] T. Aoto, K. Ito, Y. Hikosaka, E. Shigemasa, F. Penent, and P. Lablanquie. Properties of resonant interatomic coulombic decay in Ne dimers. Phys. Rev. Lett., 97, 243401 (2006).

[27] B. Najjari, A.B. Voitkiv, and C. Müller. Two-Center Resonant Photoionization. Phys. Rev. Lett., 105, 153002 (2010).

[28] A.B. Voitkiv and B. Najjari. Two-center dielectronic recombination and resonant photoionization. Phys. Rev. A, 82, 052708 (2010).

[29] A.B. Voitkiv and B. Najjari. Resonant photoionization in a system of two nonidentical atoms. Phys. Rev. A, 84,
013415 (2011).

[30] C. Müller and A.B. Voitkiv. Resonant two-photon single ionization of two atoms. Phys. Rev. Lett., 107, 013001 (2011).

[31] B. Najjari, C. Müller, and A.B. Voitkiv. Resonantly enhanced photoionization in correlated three-atomic systems. New J. Phys., 14, 105028 (2012).

[32] G. Jabbari, S. Klaiman, Y.-C. Chiang, F. Trinter, T. Jahnke, and K. Gokhberg. Ab initio calculation of ICD widths in photoexcited HeNe. J. Chem. Phys. 140, 224305 (2014)

[33] Ph.V. Demekhin, Y.-C. Chiang, S.D. Stoychev, P. Kolorenc, S. Scheit, A.I. Kuleff, F. Tarantelli, and L.S. Cederbaum. Interatomic Coulombic decay and its dynamics in NeAr following K-LL Auger transition in the Ne atom. J. Chem. Phys., 131, 104303 (2009).

[34] T. Ouchi, K. Sakai, H. Fukuzawa, I. Higuchi, Ph.V. Demekhin, Y.-C. Chiang, S.D. Stoychev, A.I. Kuleff, T. Mazza, M. Schöffler, K. Nagaya, M. Yao, Y. Tamenori, N. Saito, and K. Ueda. Interatomic Coulombic decay following Ne 1s Auger decay in NeAr. Phys. Rev. A, 83, 053415 (2011)

[35] W. Pokapanich, N.V. Kryzhevoi, N. Ottosson, S. Svensson, L.S. Cederbaum, G. Öhrwall, and O. Björneholm. Ionic-charge dependence of the intermolecular Coulombic decay time-scale for aqueous ions probed by the core-hole clock. J. Am. Chem. Soc., 133, 13430 (2011).

[36] F. Trinter, M.S. Schöffler, H.K. Kim, F.P. Sturm, K. Cole, N. Neumann, A. Vredenborg, J. Williams, I. Bocharova, R. Guillemin, M. Simon, A. Belkacem, A.L. Landers, Th. Weber, H. Schmidt-Böcking, R. Dörner, and T. Jahnke. Resonant Auger decay driving intermolecular Coulombic decay in molecular dimers. Nature, 505, 664 (2014).

[37] P. Baltzer, et al. Inner-valence states of $\mathrm{CO}^{+}$between 22 $\mathrm{eV}$ and $46 \mathrm{eV}$ studied by high resolution photoelectron spectroscopy and ab initio CI calculations. J. Phys. B, 27, 4915 (1994).

[38] F. Trinter, J.B. Williams, M. Weller, M. Waitz, M. Pitzer, J. Voigtsberger, C. Schober, G. Kastirke, C. Müller, C. Goihl, P. Burzynski, F. Wiegandt, T. Bauer, R. Wallauer, H. Sann, A. Kalinin, L.Ph.H. Schmidt, M. Schöffler, N. Sisourat, and T. Jahnke. Evolution of Interatomic Coulombic Decay in the Time Domain. Phys. Rev. Lett., 111, 093401 (2013)

[39] B. Schütte, S. Bauch, U. Frühling, M. Wieland, M. Gensch, E. Plönjes, T. Gaumnitz, A. Azima, M. Bonitz, and M. Drescher. Evidence for Chirped Auger-Electron Emission. Phys. Rev. Lett., 108, 253003 (2012).

[40] S. Bauch and M. Bonitz. Theoretical description of fieldassisted postcollision interaction in Auger decay of atoms. Phys. Rev. A, 85, 053416 (2012).

[41] R. Guillemin, S. Sheinerman, C. Bomme, L. Journel, T. Marin, T. Marchenko, R.K. Kushawaha, N. Trcera, M.N. Piancastelli, and M. Simon. Ultrafast Dynamics in Postcollision Interaction after Multiple Auger Decays in Argon 1s Photoionization. Phys. Rev. Lett., 109, 013001 (2012).

[42] A. Niehaus. Analysis of post-collision interactions in Auger processes following near-threshold inner-shell photoionization. J. Phys. B, 10, 1845 (1977).

[43] S. Sheinerman, P. Lablanquie, F. Penent, J. Palaudoux, J.H.D. Eland, T. Aoto, Y. Hikosaka, and K. Ito. Elec- 
tron correlation in Xe 4d Auger decay studied by slow photoelectron-Auger electron coincidence spectroscopy. J. Phys. B, 39, 1017 (2006).

[44] A.L. Landers, F. Robicheaux, T. Jahnke, M. Schöffler, T. Osipov, J. Titze, S.Y. Lee, H. Adaniya, M. Hertlein, P. Ranitovic, I. Bocharova, D. Akoury, A. Bhandary, Th. Weber, M.H. Prior, C.L. Cocke, R. Dörner, and A. Belkacem. Angular Correlation between Photoelectrons and Auger Electrons from K-Shell Ionization of Neon. Phys. Rev. Lett., 102, 223001 (2009).

[45] P. Burzynski, F. Trinter, J.B. Williams, M. Weller, M. Waitz, M. Pitzer, J. Voigtsberger, C. Schober, G. Kastirke, C. Müller, C. Goihl, F. Wiegandt, R. Wallauer, A. Kalinin, L.Ph.H. Schmidt, M. Schöffler, G. Schiwietz, N. Sisourat, T. Jahnke, and R. Dörner InteratomicCoulombic-decay-induced recapture of photoelectrons in helium dimers. Phys. Rev. A, 90, 022515 (2014).

[46] R. Dörner, V. Mergel, O. Jagutzki, L. Spielberger, J. Ullrich, R. Moshammer, and H. Schmidt-Böcking. Cold Target Recoil Ion Momentum Spectroscopy: a 'momentum microscope' to view atomic collision dynamics. Physics Reports, 330, 96-192 (2000).

[47] J. Ullrich, R. Moshammer, A. Dorn, R. Dörner, L.Ph.H. Schmidt, and H. Schmidt-Böcking. Recoil-ion and electron momentum spectroscopy: reaction-microscopes. Rep. Prog. Phys., 66, 1463-1545 (2003).

[48] T. Jahnke, Th. Weber, T. Osipov, A.L. Landers, O. Jagutzki, L.Ph.H. Schmidt, C.L. Cocke, M.H. Prior, H. Schmidt-Böcking, and R. Dörner. Multicoincidence studies of photo and Auger electrons from fixed-in-space molecules using the COLTRIMS technique. J. Elec. Spec. Rel. Phen., 73, 229-238 (2004).

[49] T. Havermeier, T. Jahnke, K. Kreidi, R. Wallauer, S. Voss, M. Schöffler, S. Schössler, L. Foucar, N. Neumann, J. Titze, H. Sann, M. Kühnel, J. Voigtsberger, J.H. Morilla, W. Schöllkopf, H. Schmidt-Böcking, R.E. Grisenti, and R. Dörner. Interatomic Coulombic Decay following Photoionization of the Helium Dimer: Observation of Vibrational Structure. Phys. Rev. Lett., 104, 133401 (2010).

[50] E. A. Gislason. Series expansions for Franck-Condon factors. I. Linerar potential and the reflection approximation. J. Chem. Phys., 58, 3702 (1973).

[51] W. Schöllkopf and J.P. Toennies. Nondestructive Mass Selection of Small van der Waals Clusters. Science, 266, 1345 (1994).

[52] N. Sisourat, N.V. Kryzhevoi, P. Kolorenc, S. Scheit, T. Jahnke, and L. S. Cederbaum. Ultralong-range energy transfer by interatomic Coulombic decay in an extreme quantum system. Nat. Phys., 6, 508 (2010).

[53] N. Sisourat, N.V. Kryzhevoi, P. Kolorenc, S. Scheit, and L.S. Cederbaum. Impact of nuclear dynamics on interatomic Coulombic decay in a He dimer. Phys. Rev. A, 82, 053401 (2010).

[54] W. Ackermann, et al. Operation of a free-electron laser from the extreme ultraviolet to the water window. Nature Photon., 1, 336-342 (2007).

[55] T. Shintake, et al. A compact free-electron laser for gen- erating coherent radiation in the extreme ultraviolet region. Nature Photon., 2, 555-559 (2008).

[56] K. Schnorr, A. Senftleben, M. Kurka, A. Rudenko, L. Foucar, G. Schmid, A. Broska, T. Pfeifer, K. Meyer, D. Anielski, R. Boll, D. Rolles, M. Kübel, M.F. Kling, Y.H. Jiang, S. Mondal, T. Tachibana, K. Ueda, T. Marchenko, M. Simon, G. Brenner, R. Treusch, S. Scheit, V. Averbukh, J. Ullrich, C.D. Schröter, and R. Moshammer. Time-Resolved Measurement of Interatomic Coulombic Decay in $\mathrm{Ne}_{2}$. Phys. Rev. Lett., 111, 093402 (2013).

[57] R. Santra and L.S. Cederbaum. An efficient combination of computational techniques for investigating electronic resonance states in molecules. J. Chem. Phys., 115, 6853 (2001).

[58] N. Vaval and L.S. Cederbaum. Ab initio lifetimes in the interatomic Coulombic decay of neon clusters computed with propagators. J. Chem. Phys., 126, 164110 (2007).

[59] S. Scheit, L.S. Cederbaum, and H.-D. Meyer. Timedependent interplay between electron emission and fragmentation in the interatomic Coulombic decay. J. Chem. Phys., 118, 2092 (2003).

[60] J. Itatani, F. Quere, G.L. Yudin, M.Yu. Ivanov, F. Krausz, P.B. Corkum, Attosecond Streak Camera. Phys. Rev. Lett., 88, 173903 (2002).

[61] R. Kienberger, E. Goulielmakis, M. Uiberacker, A. Baltuska, V. Yakovlev, F. Bammer, A. Scrinzi, Th. Westerwalbesloh, U. Kleineberg, U. Heinzmann, M. Drescher, and F. Krausz. Atomic transient recorder. Nature, 427, 817 (2004).

[62] M. Gensch, L. Bittner, A. Chesnov, H. Delsim-Hashemi, M. Drescher, B. Faatz, J. Feldhaus, U. Fruehling, G.A. Geloni, C. Gerth, O. Grimm, U. Hahn, M. Hesse, S. Kapitzki, V. Kocharyan, O. Kozlov, E. Matyushevsky, N. Morozov, D. Petrov, E. Ploenjes, M. Roehling, J. Rossbach, E.L. Saldin, B. Schmidt, P. Schmueser, E.A. Schneidmiller, E. Syresin, A. Willner, M.V. Yurkov. New infrared undulator beamline at FLASH. Infrared Physics and Technology, 51, 423-425 (2008).

[63] U. Frühling, M. Wieland, M. Gensch, Th. Gebert, B. Schütte, M. Krikunova, R. Kalms, F. Budzyn, O. Grimm, J. Rossbach, E. Plönjes, and M. Drescher. Single-shot terahertz-field-driven X-ray streak camera. Nature Photon., 3, 523 - 528 (2009).

[64] B. Schütte, U. Frühling, M. Wieland, A. Azima, and M. Drescher. Electron wave packet sampling with lasergenerated extreme ultraviolet and terahertz fields. Opt. Express, 19, 18833-18841 (2011).

[65] I. Grguras, A.R. Maier, C. Behrens, T. Mazza, T.J. Kelly, P. Radcliffe, S. Düsterer, A.K. Kazansky, N.M. Kabachnik, Th. Tschentscher, J.T. Costello, M. Meyer, M.C. Hoffmann, H. Schlarb and A.L. Cavalieri. Ultrafast Xray pulse characterization at free-electron lasers. Nature Photonics, 6, 852-857 (2012).

[66] U. Frühling. Light field streaking for FELs. J.Phys.B,44, 243001 (2011).

[67] http://www.pci.uni-heidelberg.de/tc/usr/icd/ICD.refbase.html 